\title{
Tendencias de Enseñanza en Redes \\ dE LA INFORMACIÓN Y La COMUNICACIÓN
}

\section{TRENDS IN EDUCATION IN INFORMATION AND COMMUNICATION NETWORKS}

\author{
Roberto Mauricio Cárdenas Cárdenas \\ Universidad Nacional Abierta y a Distancia UNAD, Bogotá, D.C., Colombia \\ Roberto.cardenas@unad.edu.co
}

Recibido: 10/06/2014 • Aprobado: 22/07/2014

\section{RESUMEN}

En el presente artículo se publican los resultados, producto de una investigación descriptiva, con relación a los procesos de enseñanza y las tendencias de estos procesos con las mediaciones tecnológicas, en lo que se ha llamado enseñanza en redes de la información y la comunicación. El estudio se orientó en dos dimensiones: la primera, tiene que ver con las modalidades de enseñanza ofrecidas a través de la red - internet-, y la segunda, con los contenidos que se publican y que forman parte de la oferta educativa de las Instituciones de Educación Superior IES, que se ha convertido en una macrotendencia en el sector educativo, más conocida como educación virtual y que para este estudio se denominará enseñanza en redes de la información y la comunicación. Por tratarse de una investigación descriptiva, la cual es definida como la caracterización de un evento, de un problema, de una situación que pueda ser sujeta a ser estudiada, para poder generar su estructura, su funcionamiento y su comportamiento, y así conceptualizar, analizar y poder ampliar el conocimiento del evento y/o situación, se realizó una indagación, tomando como referencia las páginas web de 20 Instituciones de Educación Superior en Colombia, las cuales fueron observadas y conceptualizadas a partir de las plataformas que disponen para el desarrollo de programas virtuales y/o a distancia, lo mismo que de los contenidos de los programas ofertados. Este estudio permitió confrontar la tipología de los cursos con respecto a las plataformas computacionales learning management system (LMS) y learning content management system (LCMS), como plataformas para almacenamiento y consulta de contenidos y como sistemas de producción de contenidos. Al final de este documento, se presentan propuestas y alternativas que permitirán continuar y profundizar sobre el tema de la enseñanza soportada en redes de la información y la comunicación.

Palabras clave: aprendizaje, educación, enseñanza, información, plataformas, tecnologías, virtualidad. 


\section{Abstract}

In this paper, the results product of a descriptive investigation related to the processes of education and trends of these processes with technological mediations, which has been called teaching in networks of information and communication, are published. The study was oriented in two dimensions: the first has to do with the modalities of education offered through the network - Internet -, and the second, with contents that are published and form part of the educational offer of Higher Education Institutions IES, which has become a mega trend in the education sector, better known as virtual education; for this study it will be called teaching in networks of information and communication. As a descriptive research, defined as the characterization of an event, a problem, a situation which may be subject to be studied to generate its structure, functioning and behavior, and thus to conceptualize, analyze and expand the knowledge of the event and/or situation, it was conducted an inquiry, taking as reference the web pages of 20 institutions of higher education in Colombia. These were observed and conceptualized from platforms used for the development of virtual and/or distance programs, as well as the content of the offered programs. This study allowed to confront the typology of the courses with respect to computing platforms learning management system (LMS) and learning content management system (LCMS), as platforms for storage and inquiry of contents, and as content production systems. At the end of this document, proposals and alternatives that will allow to continue and deepen the issue of education supported by the information and communication networks, are presented.

Key words: education, information, learning, platforms, teaching, technologies, virtuality.

\section{INTRODUCCIÓN}

Las instituciones de educación superior han procurado incluir en sus planes de desarrollo y en sus dinámicas administrativas y académicas, las tecnologías de información y comunicación; de hecho, muestran a sus visitantes, a sus estudiantes y colaboradores, entre otros, las bondades que tiene el uso de la tecnología en sus habituales trabajos administrativos y tareas escolares; así mismo, han generado espacios para la formación de las personas en el campo del uso de las tecnologías, tanto en el ámbito de la administración, como de la academia. Esta incorporación de tecnologías al ambiente educativo ha ocasionado una serie de cambios, relativamente poco significativos, si se contrastan con la magnitud de las tecnologías y su uso intensivo.
Es así, como un alto porcentaje de universidades y de instituciones de educación superior (IES) han estructurado en sus procesos educativos (planes de desarrollo, proyectos educativos, currículos, planes de estudio) el empleo de las tecnologías de la información y la comunicación como herramientas de apoyo a los procesos de enseñanza y de aprendizaje. Algunas, han venido empleando las tecnologías para ofrecer recursos de búsqueda y procesamiento de información; otras, como herramientas de colaboración y comunicación con los aprendices y otras han comenzado a publicar materiales educativos $\mathrm{y}$, en algunos casos, iniciado programas de cursos en línea, con el apoyo de repositorios digitales y 
objetos virtuales de aprendizaje como mediadores pedagógicos, que son considerados apoyos didácticos de la enseñanza.

Esta dinámica del sector educativo se ha convertido en una macrotendencia que mundialmente registra cambios permanentes en la dinámica educativa y, en particular, en la enseñanza, estableciendo modalidades que se ajustan a los intereses de oferta educativa por parte de las IES, como de demanda por parte de los estudiantes que ven en este segmento formativo, amplias posibilidades para su desarrollo profesional en el campo de la formación y actualización.

El segundo aspecto tratado aquí, tiene que ver con los contenidos y las plataformas sobre las cuales estos están estructurados. Para el caso de las ofertas de programas de formación en línea, se observa una amplia gama de contenidos y plataformas: de un lado, contenidos en forma de materiales de instrucción, que bien pueden ser el resultado de la tecnología educativa en forma de modelos instruccionales propios de las tendencias psicologistas del siglo pasado y que fueron aplicados en los contextos educativos primarios básicos y medios; la inspiración de esos modelos se encuentra registrada en los planteamientos productivos económicos, de un modelo que fue cuestionado en su momento $y$ que ha perdido vigencia frente a la concepción global de la economía. Se toma aquí como referencia el criterio general de los "tiempos y movimientos" [1], quienes plantearon para las empresas la función de objetivos y resultados. Esto, aplicado a la educación, se orientó bajo el criterio de la "máquina adaptativa" con el fin de establecer controles rigurosos en los procesos de enseñanza-aprendizaje, para lo cual se elaboraron paquetes de información en los que se establecieron cierto tipo de contenidos que debían ser transmitidos por los profesores en tiempos limitados y de acuerdo con unas programaciones previamente definidas y vigiladas administrativamente. Los materiales de enseñanza estructurados bajo este modelo, se han incorporado nuevamente a las oferta de formación en línea, esta vez con el mismo criterio de los "diseños instruccionales" [2].

Por otra parte, un segmento relativamente bajo de IES ha avanzado en la consideración de producir materiales de enseñanza, soportados en modelos didácticos y pedagógicos en los que se pasa de la instrucción a través de la transmisión de información, a la construcción de conocimiento a partir de la interacción que permite el uso de las tecnologías, que en forma de plataformas computacionales, ofrecen espacios de navegabilidad y usabilidad flexibles y que facilitan el desarrollo de procesos de enseñanza y de aprendizaje significativos para los estudiantes que acceden a esta modalidad de educación.

Complementando lo anterior, la investigación realizada, contempló el análisis a partir de la perspectiva de la navegabilidad y, accesibilidad. Estos términos acuñados desde lo tecnológico y desde el diseño de materiales educativos, tienen que ver generalmente con la eficacia, la eficiencia y la satisfacción de las personas cuando acceden a las plataformas para usos específicos y, en general, a la navegación por la red. Por tanto, se definen una serie de atributos cuantificables de forma objetiva; por ejemplo, el tiempo que emplea la persona en la realización de una tarea determinada, y los atributos cuantificables subjetivos, relacionados con la consecución y satisfacción en la navegación.

La accesibilidad tiene que ver, para el caso de lo educativo, con la posibilidad de acceso de todos los potenciales usuarios sin exclusión ninguna y sin restricción a las limitaciones individuales; esto implica diseñar unos modelos de producción de contenidos que permitan, precisamente, cumplir con las normas (estándares) mínimas, pero también, ampliar las esferas de uso y la navegación para sectores de la población que requieran el acceso a estos contenidos y que por razones 
de impedimentos físicos no puedan hacerlo de manera normal. En este caso, existen unos requerimientos que se denominan "pautas WAl", definidas como las pautas de accesibilidad a los contenidos de la web.

Se hace énfasis en el concepto de la inclusión, desde la perspectiva pedagógica y didáctica, lo cual tiene ciertas implicaciones en el diseño, construcción e implementación de programas de enseñanza en redes de la información; en ese sentido, se advierte la contradicción entre la adaptación del sistema al usuario y/o la adaptación del usuario al sistema. Para ello, La organización de las Naciones Unidas para la Ciencia y la Cultura UNESCO, [3] promovió la idea de una educación inclusiva y para todos, tema que fue abordado ampliamente en la Resolución de Salamanca que definió los principios y las políticas generales de la "educación para todos", y los hizo extensivos a todos los países del mundo con el fin de cerrar las brechas frente a la condición de exclusión de la educación mediada por las tecnologías.

Si bien, las modalidades de enseñanza, los contenidos digitales y las plataformas computacionales forman la triada sobre la cual se generan los programas de enseñanza en redes de la información, lo importante aquí es llegar a construir una dinámica que equilibre los tres criterios anteriores. Muy seguramente desde lo tecnológico se plantea que lo importante, el uso y la apropiación de estos medios por parte de los usuarios, debe ser comprendido desde la perspectiva pedagógica; es decir, que implique también una mirada desde la didáctica, desde el sistema de evaluación y, en general, desde la condición compleja del aprendizaje, más aún, desde el concepto del aprendizaje significativo, [4]. Este hecho es significante para la implementación de programas virtuales de enseñanza, puesto que modifica sustancialmente su estructura y determina en cierta medida la elaboración de contenidos digitales y de las mismas plataformas computacionales. Un ejemplo de lo anterior, es la plataforma Moodle (Module Object-Oriented Dynamic Learning Environment), desarrollada en código abierto, con base en el constructivismo escolar y aplicada desde la perspectiva de la inclusión, la usabilidad y la navegabilidad [5].

\section{Desarrollo de Contenidos}

Los medios educativos han variado diametralmente; los artefactos utilizados en las sesiones de clase para facilitar el aprendizaje, proyectores de diapositivas, opacos, acetatos, videos, películas, etc., hasta la incorporación del video beam, con diapositivas realizadas en el sistema de presentaciones digitales (Power Point, Freelance y otros programas similares), forman parte de la gama de apoyos para la enseñanza.

La escuela, como el profesor, entraron en la dinámica de utilizar sistemas de presentación del conocimiento de manera digital; sin embargo, estos modelos simplemente cambiaron de forma, puesto que su contenido permaneció constante. Al hacer referencia a las tecnologías, esta concepción de ayudas audiovisuales hace alusión a entornos mucho más potentes y rigurosos. Los materiales de enseñanza son tratados y considerados como mediadores pedagógicos, los cuales poseen características diferentes para cada uno de los eventos de la enseñanza.

Los materiales educativos surgen de las necesidades propias de cada una de las disciplinas; intentan solucionar de manera alternativa y emergente una situación crítica evidente en el aprendizaje de los estudiantes, aunque es necesario redefinir los criterios pedagógicos sobre los cuales se fundamente dicho material, con base en tendencias pedagógicas contemporáneas en las que se deben fundamentar las diferentes relaciones educacionales de la escuela.

Es importante destacar algunos elementos previos a la consolidación de las modalidades de enseñanza que existen en la actualidad. 
El estudio incluyó la Educación a Distancia (ED) como uno de los elementos estructurados y novedosos relacionados con el aumento de la demanda social de la educación. El fenómeno educativo de la ED fue precedido por la enseñanza por correspondencia en la que los materiales utilizados eran incipientes y se ajustaban más bien a una serie de instrucciones, lo que se conoce actualmente como paso a paso. Estos no permitían una interacción entre los estudiantes y el profesor (instructor), debido a una reducida comunicación [6]. Este tipo de sistema de enseñanza, se convirtió en alternativa para la formación de millones de personas en el mundo y se orientó hacia la enseñanza de actividades prácticas en el orden de la técnica y de oficios auxiliares.

Posteriormente y con el avance de las comunicaciones, esta tipología de enseñanza se complementó con el uso de sistemas de radiodifusión; inclusive, la televisión educativa se incorporó de una manera directa a los procesos masivos de enseñanza. Uno de estos casos es la aparición de la universidad abierta de la Gran Bretaña (Open University Britanic), que apoyaba sus clases con materiales como el video, audios, diapositivas, videocasetes, entre otros, y contaba también con el apoyo de la comunicación vía telefónica, entre los estudiantes y el tutor. En esta dinámica, se incorporaron las instituciones educativas, que ofrecían tanto programas presenciales como programas a distancia; estos últimos incorporaban la presencia del tutor (profesor) quien mantenía contacto directo con sus estudiantes a partir de visitas (clases presenciales) en lugares previamente acordados con estos. Vale la pena resaltar que una gran parte del trabajo del estudiante se desarrollaba de manera autónoma, por lo que los módulos de enseñanza se diseñaban con estrategias variadas en las que la didáctica, precisamente, desempeñaba un papel importante para su diseño y elaboración.

En general, la Educación a Distancia, según [7], ha pasado por tres generaciones:
La primera, caracterizada por la escasa y/o nula comunicación entre el profesor y el estudiante. El estudiante recibía por correspondencia los materiales impresos, la información y la orientación para procesarla. El estudiante realizaba su trabajo en solitario, enviaba las tareas y presentaba exámenes de acuerdo con una programación determinada; recibía sus notas con alguna realimentación y se preparaba para avanzar en el proceso, siguiendo las instrucciones de sus acompañantes.

En la segunda generación de la ED se implementaron otras tecnologías y se amplió la interacción, puesto que además del texto escrito, el estudiante recibía materiales audiovisuales y contaba con el apoyo del tutor, a quien podía contactar por teléfono o personalmente en las visitas programadas de acuerdo con las necesidades de los grupos o según calendarios previamente diseñados por la administración del sistema.

En la tercera generación de la ED se utilizaron materiales propios de la segunda generación y elementos mucho más sofisticados, como las tecnologías, que optimizaron el acercamiento entre los tutores y los estudiantes; este segmento de la ED se conoce como la Enseñanza Asistida por Computador (EAO) o la Enseñanza Mediada por Tecnologías (EMT).

Sin embargo, el avance y desarrollo de las Tecnologías de Información y Comunicación (TIC), ha hecho que los sistemas de enseñanza también se estén ajustando y modernizando, lo que en la actualidad se ha denominado Educación Virtual (EV).

Esta última generación de la enseñanza mediada por tecnologías EV presenta, igualmente, una serie de condiciones que han sido agrupadas de acuerdo con la metodología, las didácticas, los sistemas de evaluación y, en general, con la misma dinámica de las plataformas sobre las cuales se presentan. Sin embargo, es posible aplicar de 
manera genérica el concepto de Learning Management System (LMS) que, en otros términos, obedece a un sistema de gestión del aprendizaje. Este entra en la característica de software instalado en servidores web, el cual se emplea para diseñar, crear, almacenar, distribuir, gestionar e implementar contenidos, actividades y evaluaciones, además de permitir la colaboración entre los estudiantes y la comunicación amplia entre los estudiantes y entre los estudiantes y el profesor (tutor). El diseño de contenidos se estructura a partir de los LCMS (Learning Content Management System) los cuales permiten incorporar, organizar y sistematizar la información, así como, las condiciones por las que esta información se ajusta al grueso de los contenidos diseñados desde lo metodológico y pedagógico, para ser considerados como un material de enseñanza y de aprendizaje.

El estudio realizado permitió profundizar sobre los LMS para comprender su dinámica. Según [8], una de las funciones del LMS, con relación a los estudiantes, es la de administrar los contenidos, y hacer un seguimiento personalizado de su participación en las actividades propuestas y en la evaluación correspondiente.

En general, los LMS aplican a una metodología fundamentada en la organización didáctica (materiales, tareas, foros, chat wiki, mensajería, integración multimedia), creada por expertos disciplinares para fomentar la enseñanza y el aprendizaje en áreas determinadas del conocimiento de acuerdo con las necesidades y las condiciones del grupo de estudiantes (enseñanza a la medida).

Se destacan, como parte del estudio realizado, las características fundamentales de los LMS: interactividad, flexibilidad, escalabilidad, estandarización, accesibilidad, navegabilidad usabilidad, funcionalidad, entre otras.

El estudio incluye las modalidades que se han ido incorporando a la dinámica de la en- señanza en redes de la información, a partir de los LMS, cuyas características generales se presentan a continuación:

\section{A. E- learning o aprendizaje electrónico.}

Conocido como el sistema de enseñanza en línea, soportado con tecnologías de información, a partir del concepto de aprendizaje interactivo, flexible y accesible. Permite la conexión a la red internet, para la obtención de información y la comunicación entre el tutor y el estudiante de manera sincrónica y asincrónica; puede almacenar, mantener y administrar los materiales en un servidor web; se apoya en las tutorías y en el acompañamiento de los tutores en el proceso de enseñanza y de aprendizaje, así como, en los blogs, wikis, portafolios, chat, que forman parte de la dinámica de los cursos y/o módulos de aprendizaje.

Esta modalidad facilita el desarrollo de competencias y habilidades en el orden de la comunicación, el trabajo colaborativo, la autonomía y la autorregulación. Así mismo, el tutor establece una relación directa con sus estudiantes acompañándoles durante todo su proceso de formación.

\section{B. B- learning o aprendizaje bimodal.}

Modalidad mediada por las TIC, en la que predomina la comunicación sincrónica y asincrónica. Centra su esfuerzo en el aprendizaje (estudiante) a manera de aprendizaje autónomo y colaborativo. Aplican para este caso diseños didácticos soportados con TIC. Un ejemplo de esta modalidad es la plataforma moodle, de amplia aceptabilidad en el contexto de la educación superior ya que favorece el trabajo colaborativo a partir de encuentros sincrónicos y asincrónicos.

\section{M-learning. Aprendizaje móvil.}

También llamado aprendizaje electrónico móvil, se registra en particular por medio de los dispositivos móviles, cualquiera de ellos que tenga conectividad inalámbrica. Parte de la flexibilidad del espacio y del tiempo, y está centrado en las necesidades cognitivas del estudiante, las que 
puede soportar en gran medida por los recursos que ofrece internet, además de los registrados en forma de repositorios digitales. Los contenidos son estructurados a la medida de los dispositivos móviles, razón por la cual, la cobertura y el cubrimiento físico es ilimitado; además, hace parte de programas de formación y actualización permanente respaldados por universidades reconocidas en el mundo.

Estos sistemas de enseñanza en línea, han tenido enorme aceptación en el campo de la formación. Permiten pasar las fronteras de las lenguas y de las culturas; en este sentido, se enmarca la enseñanza virtual como una macrotendencia educativa que abre nuevos espacios para la consolidación de sistemas de comunicación. Pueden existir varias otras modalidades de enseñanza que no figuran en el espectro de las mencionadas anteriormente; sin embargo, es importante recalcar, que estas tendencias modernas de enseñanza, cada vez son más representativas en el mercado educativo del mundo y van de la mano con la evolución y desarrollo de las TIC. Es importante también considerar los modelos de enseñanza basados en modelos pedagógicos, que de una u otra manera intervienen en la construcción de diseños de contenidos aplicables a los LMS y a los LCMS, que cumplan con los objetivos de los programas, en lo relacionado con la pertinencia, cobertura y calidad, y en la dinámica de una enseñanza y de un aprendizaje permanente, continuo y significativo.

\section{Las Plataformas Y LOS CONTENIDOS}

La segunda parte de este estudio se orientó hacia el análisis de los contenidos que circulan precisamente a través de las plataformas y de las modalidades señaladas en la primera parte del trabajo. Para ello, se tomó en consideración lo relacionado con la tendencia hacia la producción de contenidos, y la relación entre las plata- formas para la enseñanza, las herramientas que disponen y la posibilidad que tienen para formular programas de enseñanza en forma de cursos. Estas plataformas se abordaron desde la perspectiva de la usabilidad y la navegabilidad; se eligieron por ser las más representativas del sector. Se tuvo en cuenta, también, lo relacionado con la producción de contenidos, enmarcados en modelos tradicionales y en modelos derivados del constructivismo escolar.

Por lo anterior, se realizó una indagación a partir de la observación de las plataformas. Se relacionaron, entonces, las herramientas vs las plataformas. Algunas de ellas cumplen con estándares internacionales, que han sido precisamente diseñadas para permitir la accesibilidad de los usuarios y su navegación a través de los contenidos. A continuación se describen las plataformas observadas:

\section{A. Dokeos. [9]}

Aplicada en sus inicios (2014) al mundo empresarial y posteriormente a los procesos de educación virtual, ha sido el resultado de un trabajo de tipo colaborativo con el soporte de los desarrolladores que aportan permanentemente a su actualización y consolidación en el campo de los LMS. Se registran en esta plataforma las amplias posibilidades para generar espacios de comunicación y de participación personal y grupal; facilita espacios para la construcción de cursos sobre metodologías activas y grupales. El código está disponible para que se pueda hacer uso del mismo o realizar adaptaciones de acuerdo con sus necesidades favoreciendo precisamente el campo de la educación.

\section{B. Chamilo [10]}

Considerada como una plataforma de acceso libre, de amplia aceptación en el sector educativo tanto a nivel básico y medio como a nivel superior; permite elaborar contenidos bajo la consideración metodológica y pedagógica del constructivismo escolar y social. Esta plataforma 
contribuye ampliamente a la generación de trabajos grupales y colectivos, puesto que dispone de herramientas que hacen posible la comunicación entre los estudiante y entre los estudiantes y el profesor, a partir de la interacción (chat, anuncios, grupos, enlaces, glosarios, wikis, ejercicios); además, cuenta con un sistema de evaluación que hace posible acciones de realimentación con los grupos de trabajo. Igualmente, permite el manejo de videoconferencias y el acompañamiento a los usuarios, lo mismo que su evaluación mediante la obtención de informes completos de sus actividades y la graficación de estos. Es de licencia GNU/ GLP (software libre) lo cual permite libertad para usar, modificar, mejorar y distribuir contenidos.

\section{Claroline [11]}

Plataforma LMS, reconocida por su amplia versatilidad y facilidad para el montaje de cursos en línea y como apoyo a los sistemas de formación presencial. Se pueden publicar contenidos en forma de cursos en varios formatos de archivo y permite ampliamente la comunicación entre los estudiantes, favoreciendo la creación de grupos de trabajo y facilitando sus envíos a través de las herramientas de comunicación, sin límite de usuarios. Utiliza el lenguaje de programación PHP y MySQL como SGBD. Sigue las especificaciones de SCORM e IMS.

\section{ATutor [12]}

Es considerado como un LMS de acceso libre; permite que los productores de contenidos puedan diseñar, ensamblar y publicar contenidos educativos como soporte a sus clases, tanto en ambientes tradicionales de enseñanza como en ambientes mediados por TIC. Facilita la producción de contenidos y permite modificarlos de acuerdo con las necesidades de los estudiantes; genera espacios de comunicación. Utiliza un servidor Apache, con motor de base de datos MySQL. Trabaja sobre plataformas Windows, GNU/Linux, Unix, Solaris y tiene soporte en 32 idiomas. Para su instalación se necesita un servidor en el cual se encuentre previamente instalado PHP y MySQL, y bajar el archivo instalador, procedimiento orientado por el grupo de estudios e investigación sobre influencia de TIC en educación.

\section{E. Moodle [13]}

Es otra de las aplicaciones de código abierto (código libre) de una amplia aceptación en el contexto de la educación en un alto porcentaje de países. Puede ser copiada, usada o modificada, siempre que sean públicos los cambios registrados. Lo relevante de esta plataforma es que ha sido diseñada bajo principios de constructivismo escolar y social; en ella se observa una amplia gama de herramientas muy útiles para los profesores ya que permiten la comunicación permanente a través de foros, blogs, wikis, chats, con un amplio manejo por parte del profesor frente a los contenidos y las actividades propuestas en los módulos de aprendizaje. Permite el acceso tanto de administradores, como de tutores y estudiantes, de manera independiente, lo que facilita el rol del profesor como acompañante del proceso.

\section{F. Sakai, [14]}

LMS construido a partir de un proyecto de investigación de las Universidades de Indiana, Michigan, MIT y Stanford. Esta plataforma está orientada hacia el fortalecimiento de las actividades relacionadas con la construcción de materiales por parte de los estudiantes, lo que se conoce como trabajo autónomo y colaborativo orientado por metodología basada en el portafolio. Se diferencia por disponer de herramientas generales de colaboración, de enseñanza y de aprendizaje, de administración y de portafolio, lo que facilita los procesos de enseñanza y permite su evaluación permanente y detallada.

\section{G. LRN [15]}

Es reconocido como un sistema dinámico de portales que facilita la creación de contenidos, lo mismo que su administración y la administración de herramientas colaborativas. Ampliamente utilizado por aquellas personas que pertenecen a redes y comunidades de aprendizaje, 
permite la elaboración de encuestas, foros, noticias, calendarios cuestionarios, exámenes, encuestas, blogger, entre otras herramientas, que facilitan la comunicación y la circulación de documentos e información, con el fin de que las comunidades mantengan relaciones permanentes y actualizadas.

En este orden de ideas, también se realizó la observación de varias LMS de tipo comercial más significativas, como:

\section{H. Almagesto [16]}

Sistema de producción de contenidos, utilizado en el sur del continente americano, cuenta con amplia acogida por tratarse de un producto licenciado y por sus características de estabilidad, operabilidad y seguridad. Se caracteriza por herramientas orientadas hacia el control de los procesos de enseñanza. Tiene en su estructura: aulas virtuales, aulas de exámenes, aula de autoevaluación, salas de debate, foros de debate, conferencias en línea y videotecas, entre otros.

\section{l. Blackboard [17]}

Es una de las plataformas, quizá, más utilizadas en el sector educativo. Está conformada por módulos de contenidos, herramientas de comunicación, de evaluación de seguimiento y de gestión del aprendizaje. Presenta un ambiente virtual constituido por cuatro áreas primarias: administración de contenidos, comunicación, evaluación y control. Esta última, permite que los profesores tengan la información de todos y cada uno de los estudiantes y el acceso directo a las evaluaciones, facilitando planificar el tiempo de las actividades y la información personal de los estudiantes.

\section{J. E-ducativa [18]}

Es una de las plataformas orientadas hacia el fortalecimiento de estructuras comunicativas y el intercambio de experiencias en el orden educativo y académico; se puede manejar como un espacio colaborativo que cuenta con un alto nivel de interactividad desde lo asincrónico.
Permite la construcción de redes académicas que formulen problemáticas comunes para que a partir de la interacción planteen soluciones en lo que se ha llamado comunidades de aprendizaje. Se aplica también esta misma dinámica a la comunicación en el orden educativo permitiendo la elaboración de proyectos y sistemas de evaluación por portafolios.

\section{K. Nixty [19]}

Es una plataforma que se construyó a partir de los principios funcionales de blackboard y linkedin. Provee espacios para la creación de cursos virtuales y ofrece una variada gama de cursos, algunos gratuitos, que están abiertos a la comunidad. El mismo sistema se encarga de desarrollar campañas de publicidad sobre los cursos montados para lo cual genera unos cobros que están en los parámetros internacionales de costos por estos servicios.

\section{Edu2.0 [20]}

Es una plataforma mixta. Tiene una serie de herramientas gratuitas, pero las significativas, tienen costos para el usuario, Facilita la comunicación y el tránsito de información ya que se adapta a los dispositivos móviles y se puede acceder desde estos, lo que permite el desarrollo y la comunicación de sistemas de enseñanza $m$-learning. Se adecúa fácilmente a las tareas escolares pues dispone de herramientas para la creación de contenidos, evaluación del aprendizaje, seguimientos y realimentaciones; dispone de espacios para que los padres de familia tengan información acerca del progreso de los estudiantes y de los mensajes que la institución envíe sobre el rendimiento académico de los mismos.

\section{Contenidos Digitales}

Luego de la revisión de las plataformas LMS y LMCS, se abordó el tema de los contenidos digitales. Para este estudio se observaron programas académicos ofertados por instituciones 
educativas de formación superior, de manera aleatoria, se consideraron aquellas que tienen mayor visibilización en internet, precisamente por la oferta de programas académicos en modalidad virtual. Por tratarse de una observación externa direccionada a la condición y caracterización de los cursos y módulos, se omite el nombre de las instituciones. Para esta observación se tuvieron en cuenta dos criterios: pedagógico y didáctico. Uno, el relacionado con la estructura, organización y metodología, a partir de los diseños instruccionales enfocados en el instruccionismo y que tienen las características de la pedagogía tradicional centrada en los contenidos y la información. Y en un segundo aspecto, la observación a partir de modelos emergentes en los que se incluye sustancialmente el constructivismo escolar y social con sus características metodológicas y didácticas.

Se tomaron como referencia una muestra de 20 Instituciones de Educación Superior IES, y se analizaron 20 cursos ofertados de acceso libre, entre los cuales se delimitó la observación a 20 módulos, respectivamente.

De acuerdo con las dos variables iniciales (Modelo instruccional - Modelos alternativos), se generó un modelo comparativo que arrojó como resultado que un $87 \%$ de los cursos analizados obedece a un esquema de diseño instruccional. Este consiste en centrar la actividad académica en la transmisión de información y de contenidos, a partir de la metodología de objetivos y resultados. Aquí se observa que el proceso de enseñanza se hace a través de una serie de documentos y presentaciones (pdf, Word, ppt, prezi, cmap-tools), que deben ser leídos por el estudiante, para luego responder quices y exámenes, los cuales han sido diseñados previamente por los llamados contenidistas, entregando de manera automática la evaluación y su resultado en forma numérica. Los cursos son fortalecidos por videos, audios, narraciones; y en el orden de la comunicación, presentan encuentros sincrónicos o asincrónicos que con antelación están programados. En algunos casos, se presenta el curso por proyectos; sin embargo, las pautas para el desarrollo de los mismos están previamente consideradas y se ajustan a los contenidos. Finalmente, son cursos de corta duración (6 a 10 semanas) que se registran en créditos que van siendo sumados para la obtención de certificaciones finales. Cabe anotar que estos cursos reposan en su mayoría en plataformas LMS comerciales.

De otro lado, solo el $15 \%$ de los cursos observados presenta criterios metodológicos emergentes y asociados al constructivismo escolar y social. Se trata aquí de incorporar modelos pedagógicos y didácticos aplicados a la enseñanza de saberes específicos, por lo que se formulan diseños centrados en el estudiante a partir de proyectos de investigación y proyectos con base en sus necesidades (problemas propios del entorno escolar y laboral), para ser tratados por medio de lecturas críticas que se ajustan a procesos de orden investigativo y problémico. Estos cursos se alojan en plataformas no licenciadas, como moodle, cuyas características, como LMS, permiten adaptarse a este tipo de modelo de enseñanza que se aproxima a la enseñanza y el aprendizaje significativo por proyectos y con enfoques colaborativos y problémicos. En cuanto a la evaluación, se incorporan elementos novedosos como las competencias, el portafolio, y los sistemas de acompañamiento por parte del tutor que se hace de manera abierta a partir de los foros, wikis, blogs y sistemas de comunicación sincrónica apoyados en las redes sociales.

\section{Resultados y Análisis}

A partir de los resultados obtenidos mediante un proceso metodológico asociado a las características de una investigación descriptiva 


\section{Conclusiones y TRABajos Futuros}

centrada en la interpretación y análisis de la ginas web de 20 IES y de la información obtenida en las publicaciones sobre el tema de la enseñanza en redes de la información y la comunicación, se puede afirmar que existe en la actualidad una fuerte tendencia por parte de las IES, a la incorporación de sistemas de formación con base en cursos de corta y mediana duración, lo mismo que de programas académicos formales de pregrado y posgrado, lo que se conoce como educación virtual y recientemente como "educación transfronteriza" [21]. Esta tendencia ha generado la proliferación de programas y de ofertas académicas en el mundo entero y se muestra como la evolución de la educación a distancia, como se mencionó al inicio de este documento.

Estos sistemas de enseñanza soportados con las herramientas tecnológicas de comunicación e información han tenido también gran aceptación en aquellas personas que por su ubicación física, por sus limitaciones de tiempo y de espacio, no pueden acceder a los programas tradicionales y presenciales de formación. Por tanto, se cuenta con modalidades (learning) que se ajustan a los requerimientos técnicos, tecnológicos y metodológicos que las instituciones requieren para difundir estos programas.

De otra parte, se observó una tendencia a la utilización de plataformas LMS y LMCS, no licenciadas, pero combinadas con plataformas licenciadas, con el fin de garantizar, en cierta medida, la operabilidad y el soporte técnico que requiere el sistema. Así mismo, el estudio arroja que los modelos convencionales- tradicionales de enseñanza prevalecen en el sector educativo, debido a la transposición del modelo instruccional propio de la pedagogía tradicional centrada en los contenidos, que facilita el control y la administración de los programas académicos.
A manera de conclusiones se pueden plantear las siguientes:

- Se registra tanto en el ámbito nacional como en el internacional, un alto crecimiento de la oferta de programas académicos virtuales y/o a distancia, soportados por tecnologías de la información y comunicación.

- Se consideran las modalidades e-learning, b-learning, $m$-learning, como los espacios para ofrecer los programas académicos ajustados a las necesidades de formación tanto del país como del ámbito internacional.

- El estudio observó la consolidación de los MOOCs (cursos abiertos masivos en línea), como el programa que ha tenido mayor aceptación y visibilidad en el sector educativo.

- El análisis de los programas y cursos ofertados se centra en sistemas de enseñanza tradicional en los que prevalecen los modelos instruccionales en alto porcentaje.

- Los cursos, módulos, con características alternativas por su metodología son relativamente bajos y se centran en modelos problémicos y por proyectos, complementados con sistemas de evaluación y comunicación sincrónica y asincrónica.

- Existen vacíos conceptuales frente a la consideración pedagógica de los programas, ya que a pesar de tener un soporte didáctico (repositorios digitales) amplio en la red, los cursos no reflejan el uso y la apropiación de estas herramientas. 
- Los sistemas de evaluación del aprendizaje siguen siendo limitados y reflejan el modelo de la enseñanza presencial, tanto en lo numérico como en lo conceptual.

Aunque existe variada documentación sobre el tema de la enseñanza en redes de la información y la comunicación, se hace necesario continuar con los estudios sobre este tema y en particular sobre aquellos elementos que forman parte sustancial de los procesos de enseñanza y de aprendizaje. Consideraciones pedagógicas, metodológicas, didácticas, evaluativas deben ser motivo de estudios complementarios, que permitan visualizar nuevos escenarios educativos, en los que se transformen los procesos orientados hacia la consideración de una educación acorde con el desarrollo de las sociedades y con la evolución de las mismas. Por ello, es indispensable continuar profundizando en este campo y en particular en las siguientes problemáticas:

- Los procesos educativos centrados en el aprendizaje.

- Modelos didácticos soportados en tecnologías de la información y comunicación.

- Sistemas de aprendizaje fundamentados en procesos y proyectos.

- Modelos de evaluación del aprendizaje correspondientes a las dinámicas pedagógicas contemporáneas.

- Estudio sobre plataformas LMS y LMCS adaptables y modificables de acuerdo con los programas y cursos ofertados por las IES.

- Permanencia de los estudiantes en sistemas de enseñanza de corte virtual y/o a distancia con soporte de TIC.

- Procesos de comunicación en redes de la información y la comunicación a partir de las redes sociales como elemento globalizador.

\section{REFERENCIAS}

[1] [A. Barba Alvarez, Frederick Winslow Taylor y La Administración científica: contexto, realidad y mitos, México: Unam, 2010.

[2] L. A. Caro B, Concepciones Educativas Contemporáneas, Bogotá: Kapra Editores, 2006.

[3] UNESCO, EDucación Inclusiva para Todos, Mexico: Unesco, 1990.

[4] D. Ausubel, J. Novak y H. Hanesian, Educational Psychology: A Cognitive View, New York: Rinehart \& Winston, 1978.

[5] M. y. T. Dougiamas, Interpretive analysis of an internet-based course constructed using a new courseware tool called Moodle. Proceedings of the Higher Education Research and Development, Perth: Curtin University of Technology, 2002.

[6] L. Sauvé, Pour une éducation relative à l'environnement., Montreal: Guérin, 1997.

[7] S. Nipper, Third generation distance learning and computer conferencing, Oxford: In R. Mason \& A. Kaye (Eds.), 1989.

[8] L. Álvarez, A Learning Activities Authoring Tool for IGUAL Project. Proceedings of 1st International Workshop on Open Technology Transfer and Learning Solutions for Programming Education, Bucharest: University of Civil Engineering, 2012.

[9] Dokeos, «http://www.dokeos.com/,» [En línea]. Available: http://www.dokeos.com/. [Ültimo acceso: 12 Abril 2016].

[10] https://chamilo.org/, «https://chamilo.org/,» [En línea]. Available: https://chamilo.org/. [Último acceso: 23 Abril 2016].

[11] http://www.claroline.net/, «http://www.claroline.net/,» [En línea]. Available: http://www.claroline.net/. [Último acceso: 12 Abril 2016].

[12] http://atutor.ca/, «http://atutor.ca/,» [En línea]. Available: http://atutor.ca/. [Último acceso: 12 Mayo 2016].

[13] https://moodle.org/?lang=es, «Moodle,» [En línea]. Available: https://moodle.org/?lang=es. [Último acceso: 17 Mayo 2016].

[14] http://www.sakaiproject.org/, «http://www.sakaiproject.org/,» [En línea]. Available: http://www.sakaiproject.org/. [Último acceso: 22 Mayo 2016].

[15] http://www.learningnurse.org/, «http://www.learningnurse.org/,» [En línea]. Available: http://www.learningnurse.org/. [Último acceso: 6 Mayo 2016].

[16] http://www.almagesto.com/, «http://www.almagesto. com/,» [En línea]. Available: http://www.almagesto. com/. [Último acceso: 15 Abril 2016].

[17] http://www.blackboard.com/International/LAC. aspx?lang=en-us, «http://www.blackboard.com/International/LAC.aspx?lang=en-us,» [En línea]. Available: http://www.blackboard.com/International/LAC. aspx?lang=en-us. [Último acceso: 15 Abril 2016]. 
[18] http://www.educativa.com/, «http://www.educativa. com/,» [En línea]. Available: http://www.educativa. com/. [Último acceso: 11 Abril 2016].

[19] http://nixty.com/, «http://nixty.com/,» [En línea]. Available: http://nixty.com/. [Último acceso: 30 Marzo 2016].

[20] https://www.edu20.org, «https://www.edu20.org,» [En línea]. Available: https://www.edu20.org. [Último acceso: 29 Marzo 2016].
[21] https://books.google.com/books?isbn=970704070X, «https://books.google.com/books?isbn=9707040 70X,» [En línea]. Available: https://books.google. com.co/books? id=Zh09X80odyoC\&pg=PA3 $9 \& \mathrm{lpg}=\mathrm{PA} 39 \& \mathrm{dq}=$ educaci $\% \mathrm{C} 3 \% \mathrm{~B} 3 \mathrm{n}+$ transfron teriza\&source $=$ bl\&ots $=v 4 n 5$ NtygjK $\&$ sig $=6 \mathrm{P} 7 \mathrm{oN}$ WYn2dbLKmccrKutBKcNWLI\&hl=es \&sa=X\&re dir_esc $=y \# v=$ onepage $\& q=$ educaci $\% C 3 \% B 3 n \% 20$ transfronteriza\&f=false. [Último acceso: 30 Marzo 2016]. 
